\title{
Simulation of the progression of yellow spot on wheat using a functional-structural plant model (FSPM): Model concepts
}

\author{
K. Streit $^{\text {a }}$, C. Bahr ${ }^{\text {a,b }}$, J.B. Evers ${ }^{\text {c }}$ and M. Renton ${ }^{\text {a }}$ \\ ${ }^{a}$ Schools of Biological Sciences, Agriculture \& Environment, University of Western Australia, Crawley, WA \\ 6009, Australia \\ ${ }^{b}$ Department Ecoinformatics, Biometrics and Forest Growth, University of Göttingen, 37077 Göttingen, \\ Germany \\ ${ }^{c}$ Centre for Crop Systems Analysis, Wageningen University, 6708, PB, Wageningen, The Netherlands
}

Email: katarina.streit@uwa.edu.au

\begin{abstract}
Despite disease control management, each year part of crop harvest is lost due to plant diseases. Yellow spot is an important foliar wheat disease throughout the world. The fungus that causes the disease survives on wheat stubble and this is most commonly the source of primary infection (by ascospores) in a crop canopy in the next season. On infected leaves, lesions are formed, surrounded by yellow halos. After a latency period, conidia, the cause of secondary infection, are produced on lesions and are spread over long distances by wind. The secondary cycle can repeat several times through the season and results in the progression of the disease in the canopy. Weather conditions and the developmental stage of the crop play an important role in the progression and severity of disease in the crop canopy.
\end{abstract}

To study the interactions between pathogen, climatic conditions and growing host crop, we developed an epidemiological model of Pyrenophora tritici-repentis, the fungal pathogen that causes yellow spot, and coupled it with an existing functional-structural plant model (FSPM) for cereal crops. An FSPM simulates mutual interactions between plant architecture (structure) and physiological processes (function) in plants at a (sub)organ scale, affected by environmental conditions. In our model, light interception and temperature determine the development and the growth of the cereal crop. Temperature, rainfall, relative humidity and wind data control the development of yellow spot. The pathogen submodel predicts maturation of ascospores and simulates production and wind dispersal of conidia across the canopy. Conidia are transported inside a virtual cone starting from a sporulating lesion and with the axis following the wind direction.

Simulations demonstrated horizontal and vertical progression of the disease in the growing crop canopy. However, the upper leaves grew often away from the disease after the begin of stem elongation. In the future we will perform enhanced sensitivity analysis that should help us to identify the most (least) important parameters and so help in the process of model parameterisation. Epidemiological models coupled to models for plant architecture and growth under different climatic conditions are a promising tool to study the dynamics of plant-pathogen-environment interactions and their effect on crop yield. Furthermore, the coupled model can be used as a simulation tool to study the impact of different disease management approaches and lead to improved disease control. We will test the applicability of the model against field data on disease progression in spring wheat.

Keywords: Functional-structural plant model (FSPM), disease progression, yellow spot, wheat, wind dispersal 


\section{INTRODUCTION}

Each year, part of global harvest (10-16\%) is lost due to plant diseases and additional postharvest losses (6-12\%) (Chakraborty and Newton, 2011). Plant disease control has an important role in improving food security in the context of increasing population and climate change (Chakraborty and Newton, 2011). In Australia, the loss of the average annual value of wheat crop from diseases was $19.5 \%$, with yellow spot dominating the loss $(23 \%$ of the total loss; Murray and Brennan, 2009). These numbers consider three methods of disease management: use of resistant cultivars, paddock preparation and management, and application of pesticides; without the disease control, the potential losses would be higher (Murray and Brennan, 2009).

Yellow spot of wheat is caused by a necrotrophic fungus Pyrenophora tritici-repentis, and disease development is strongly related to temperature and moisture conditions. The fungus survives on wheat stubble from the previous season. Black fruiting bodies (pseudothecia) on the wheat residue produce sexual spores (ascospores), which are the cause of primary infection in a crop canopy in the next season. Rainfall and temperature favour thematuration of ascospores (Summerell and Burgess, 1988; Galloway et al., 2017) and a combination of environmental conditions triggers their release. Dispersal is local, over a short distance (Rees and Platz, 1980; De Wolf and Francl, 1998) and is supported by wind. On infected leaves, the fungus produces oval to diamond-shaped lesions, surrounded by yellow halo (De Wolf and Francl, 1998). Temperature and moisture influences the production of asexual spores (conidia), the cause of secondary infection (Francl, 1998). Conidia are then dispersed large distances by wind (Francl, 1997). Under favourable climatic conditions, the secondary cycle repeats several times in a lifetime of the disease and results in the rapid development of the disease in the growing crop (Rees and Platz, 1980).

There have been many efforts to model disease development for different crops. De Wolf and Isard (2007) reviewed a group of disease prediction models looking at the components of disease life cycle (associated with environmental conditions) included in the models. The disease life cycle can be characterized by a series of stages: dormancy, reproduction, dispersal and pathogenesis (De Wolf and Isard, 2007). The progression through these stages is determined by the interactions between pathogen, host and environment (De Wolf and Isard, 2007; De Wolf et al., 1998). Previously, artificial neural networks were used to predict infectious periods of yellow spot, from the crop growth stage and a set of environmental variables, and their prediction accuracy was higher than by logistic regression models (De Wolf and Francl, 1997). Although this kind of modelling approach can identify the relationships between environmental variables and disease development, it does not allow a study of how pathogen dynamics is linked to plant architecture and growth. Changes in plant architecture and growth affect disease progress and severity (Ando et al., 2007; Wilson and Chakraborty, 1998). Therefore, coupling epidemiological models with models for plant architecture and growth under different climatic conditions can help to better understand the dynamics of plant-pathogen-environment interactions and lead to improved disease control.

Several recent host-pathogen models accounted for plant architecture and/or growth, with the host plant submodel considered at different levels of detail (Calonnec et al., 2013; Caubel et al., 2012). A plant submodel can be represented, e.g. 1) by assuming total leaf area, 2) as crop model (including growth and different organs or organ compartments, but not explicit architecture; Caubel et al., 2012; Milne et al., 2003), 3) using structural models (including 3D plant architecture and development; Calonnec et al., 2008; Pangga et al., 2011; Landry et al., 2017), or 4) using functional-structural plant models (FSPMs) (Robert et al., 2008; Garin et al., 2014). The latter represent the class of the most complex models, which aim at simulating interactions between 3D plant architecture (structure) and physiological processes (function) in plants, as well as environmental conditions. With FSPMs coupled in host-pathogen models it is possible to study and test different hypotheses related to the interactions between disease development, plant architecture and growth, environment, and even management decisions in one model (Pangga et al., 2011; Calonnec et al., 2013).

For yellow spot on wheat, Galloway et al. (2017) observed that in different locations in the Western Australian Grainbelt (from the southern to the northern cropping areas) with different climatic conditions, the risk of disease development varied, as the rate of pseudothecia maturation, the date of crop emergence and the progress of secondary infection differed. To analyse the interactions affecting the progression of yellow spot, in this study, we aimed to develop a model for its life cycle and couple it with an existing FSPM model for cereal crops, as we were not aware of such model published previously. In the following sections we will present the main principles of the model.

\section{MODELLING APPROACH}

\subsection{Model overview}

The model couples crop growth and development, driven by light interception and temperature, with the development of yellow spot, driven by temperature, rain, relative humidity and wind data. To model the crop 
growth, including its architecture in 3D over time, we use an FSPM that was developed for cereal crops, with a daily time step (Evers and Bastiaans, 2016). We modified the FSPM to work with leaf segments rather than with a leaf as a whole, as previously. Among existing FSPMs for cereal crops, or wheat in particular, we chose this model because it simulates plant growth in a mechanistic, rather than a descriptive manner (described below).

The newly developed pathogen submodel considers the following substages of the disease cycle: 1) reproduction: maturation of ascospores, 2) pathogenesis: infection (primary infection by ascospores and secondary infection by conidia) and latency period, 3) dispersal: transport and deposition of conidia. The pathogen submodel is based on principles from other host-pathogen models (Calonnec et al., 2008; Robert et al., 2008; Garin et al., 2014; Caubel et al., 2012), adapted and extended for yellow spot. We derived the parameter values based on literature review and from previous (unpublished) experiments. We implemented the model in the GroIMP modelling platform (Hemmerling et al., 2008).

\subsection{Effect of environment}

The model takes into account several environmental variables which are known to be important in controlling plant growth and development, and the pathogen life cycle (Figure 1):

- Plant growth and development: latitude, day length, incoming radiation, temperature (daily values),

- Pathogen life cycle: temperature, rain, relative humidity (RH), wind speed and direction (daily or hourly values).

Day length and the intensity of incoming light are computed according to Goudriaan and van Laar (1994), based on the site latitude and day of year. The light comes from a dome and an arc of light sources representing the sky and the daily course of sun, respectively (Evers et al., 2010; Evers and Bastiaans, 2016), placed around a virtual canopy. We use a Monte Carlo path tracing method (Hemmerling et al., 2008) to compute the amount of light absorbed by the leaves. The remaining environmental variables are extracted from daily or hourly meteorological data.

\subsection{Canopy development}

The crop development is described by rewriting rules (Hemmerling et al., 2008) and is temperature-driven (Evers and Bastiaans, 2016). Leaves, which can bear lesions, are modelled as a sequence of rectangular segments (30 to 60 depending on leaf size) with a geometry typical for gramineous leaves. The size of the leaf depends on the amount of assimilates allocated to it, which is driven by both production of assimilates by leaves, and the demand for assimilates by growing leaves and other organs.

Leaf carbon assimilation is computed from absorbed radiation using a negative exponential photosynthesis-light response model. Leaves produce new assimilates until they reach a certain age or if the light absorbed by a leaf is below a given threshold. Organ demand for assimilates is modelled using a relative sink strength concept (Marcelis, 1996). A fraction of available assimilates for growth is assigned to each organ based on its sink strength relative to the total plant sink strength, and taking into account growth and maintenance respiration. Sink strength is defined as a potential demand at a given organ age, expressed as thermal time, and computed as a derivative of a beta growth function (Yin et al., 2003). Any remaining assimilates are stored in a common pool, for use in the next model time step. Individual plants are arranged into a canopy (Figure 2).

\subsection{Life cycle of the pathogen}

Maturation of pseudothecia producing ascospores is modelled as a consequence of synchronous rain events and favourable temperature, over a given preceding time period (Galloway et al., 2017; Salam et al., 2003). After a number of suitable days, ascospores are released. The continuous discharge of ascospores over the season (Galloway et al., 2017) and the effect of weather on the release are currently not considered. The first predicted occurrence of mature ascospores is used as input for primary infection on a random seedling leaf segment and the starting point of the pathogen submodel.

Lesions on leaf segments grow linearly from their initial size using a fixed growth rate based on thermal time, similarly to Robert et al. (2008). For simplification, they have a circular shape and their size (radius) is limited by the available leaf green area. After a fixed latency period following an infection, sporulation occurs. The quantity of produced conidia is a function of lesion area (Francl, 1998). Conidia are transported by wind, taking wind direction in afternoon hours (Francl, 1997) as a default setting. The wind speed is not considered as a limiting factor for release and transport of spores.

Secondary infection occurs after favourable conditions of moisture and temperature, with $\mathrm{RH}$ above $90 \%$ (a simplified model of leaf wetness) and temperature between 15 and $28^{\circ} \mathrm{C}$ for the duration of at least 6 hours (M. Shankar, personal communication). Following Calonnec et al. (2008), the probability that a spore will cause 
secondary infection depends on temperature and leaf age factor, with the maximum infection rate at optimal temperature and decreasing with leaf age. A spore will not cause a new infection on a senesced leaf.

\subsection{Wind dispersal}

The released conidia are transported in the direction of the wind within a cone characterized by a half opening angle $\theta$ and extending to infinity. We adapted the cone representation from previous host-pathogen models which considered plant architecture in 3D (Garin et al., 2014; Calonnec et al., 2008). The tip of the cone is located at a sporulating (source) lesion and its central axis has the direction of the wind. The conidia released from a source lesion will be distributed among all the leaf segments lying inside the cone. The number of spores deposited at a leaf segment is computed according to Calonnec et al. (2008). It is proportional to the area of the leaf segment, and decreases exponentially with the distance from the cone tip and linearly with the distance from the cone axis.

\section{RESULTS}

\subsection{Model setup}

The simulations were run for weather data from 2013 from Eradu (Figure 1), an example of the northern cropping area in the Western Australian Grainbelt. Plants were placed in rows in a grid, with $25 \mathrm{~cm}$ row spacing and $2.5 \mathrm{~cm}$ plant spacing (Figure 2). The simulation of crop growth started on 1 May 2013, with (successful) primary infection on 24 May 2013.

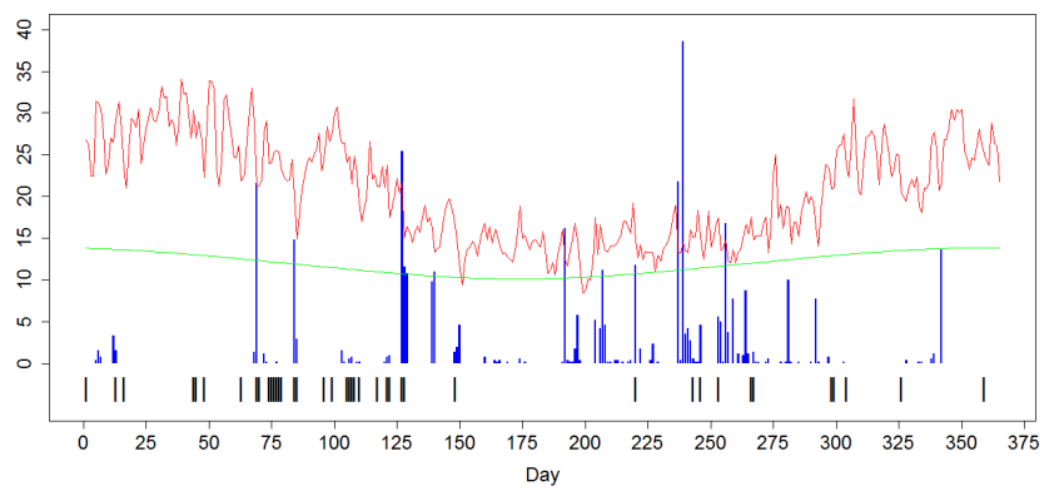

Figure 1. Daily environmental data used for simulations: air temperature $\left({ }^{\circ} \mathrm{C}\right.$, red) and rainfall (mm, blue) are taken from weather data. Day length (h, green) was simulated according to Goudriaan and van Laar (1994). The days favourable for infection (black bars) computed from hourly data, are those with RH above $90 \%$ and air temperature between 15 and $28^{\circ} \mathrm{C}$ for at least 6 hours.

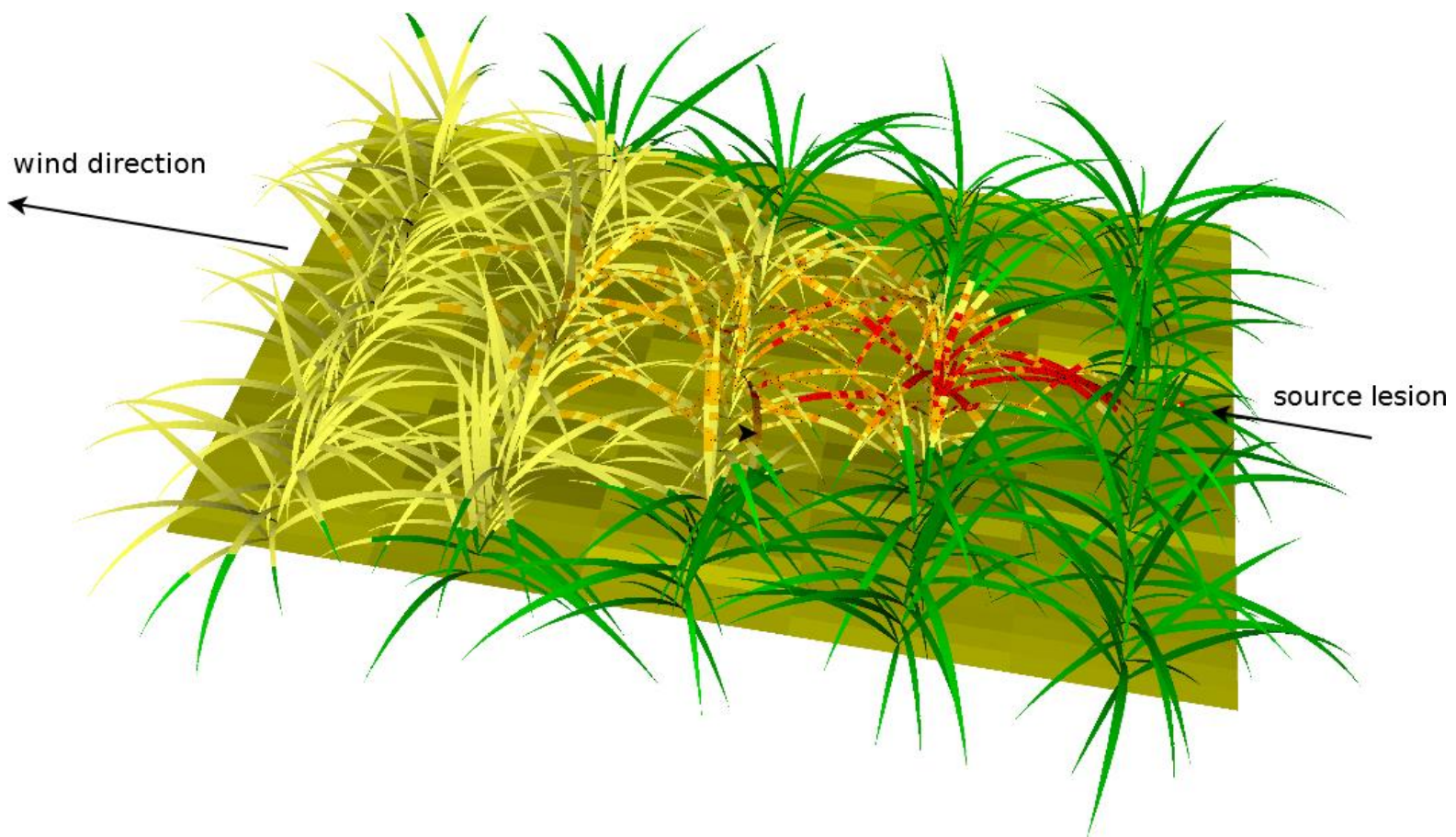

Figure 2. Schematic example of canopy setup (grid with 5x30 plants) and dispersal in young canopy from one source lesion after one wind event. The source was placed on a randomly selected leaf segment. Conidia were transported inside a virtual cone (with the opening angle of $22.5^{\circ}$ ), whose tip was located at the source lesion. Leaf segments lying inside the cone are coloured and the colour gradient represents the number of deposited conidia (shown as black dots) on leaf segments (from yellow - no conidia, to red - highest conidia count). 


\subsection{Wind dispersal}

Figure 2 shows an example of the conidia transport and deposition on leaf segments from one source lesion after one wind event. The colour gradient on the leaf segments lying inside the imaginary cone demonstrates that the number of deposited spores decreases with the distance from the source lesion, as well as from the cone's axis (from red to yellow).

\subsection{Disease progression}

Figure 3 demonstrates the disease progression in a growing canopy. The model computes the appearance of new leaves as a function of thermal time. The initial inoculum is placed on one selected segment. Conidia are deposited on existing leaf segments based on the 3D location of the segments. Within a segment, conidia are placed randomly. Several stochastic components in the model have impact on the disease progression in the canopy, e.g. plant/leaf orientation. Transport of conidia in a cone shape combined with dynamic plant growth and repeated disease cycle results in the horizontal and upward conidia spread and disease progression.

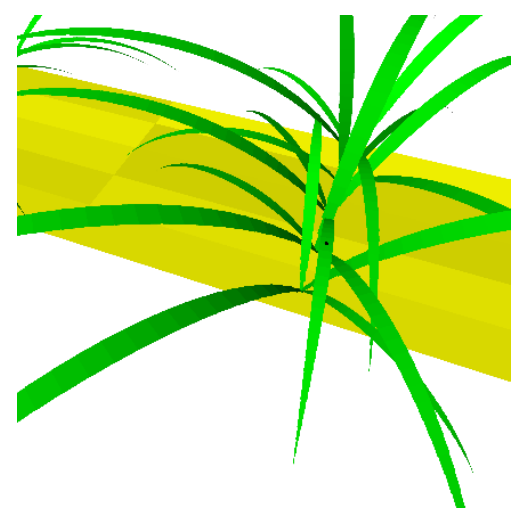

a

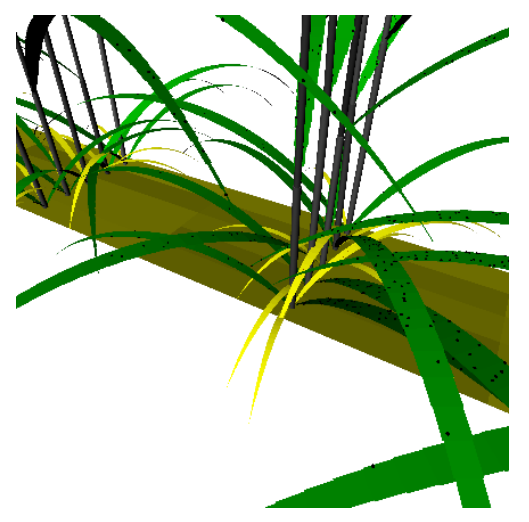

b

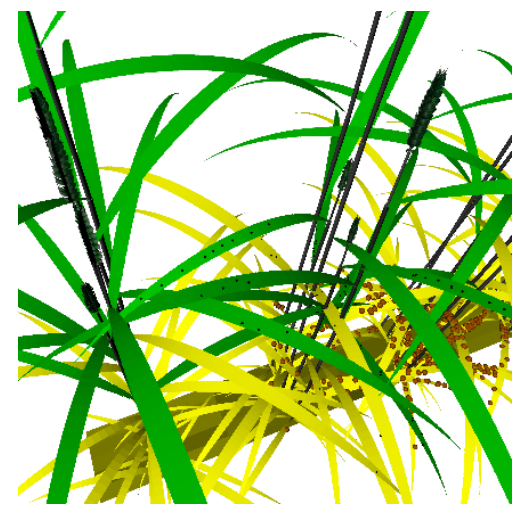

c

Figure 3. Simulated progression of the disease: a) primary inoculum on a seedling, b) released conidia are transported by wind and deposited on leaf segments, c) under favourable condititions, plant growth and repeated disease cycle result in an upward spread. Black dots refer to deposited spores which will develop into lesions. Brown colour refers to lesions that have already produced new spores.

\section{DISCUSSION AND CONCLUSIONS}

We developed a model of the progression of yellow spot from arbitrarily infected seedling leaves in the canopy. The model considers several substages of the disease cycle, including primary and secondary infection, and the spread of conidia by wind. The growth of spring wheat was mimicked using an FSPM for cereal crops.

Simulations demonstrated horizontal and vertical progression of the disease in the example setup. Often, the upper leaves grew away from the disease after the stem elongation started. This can probably be explained by the use of a simplified wind dispersal inside a virtual cone and by considering a finite canopy. The disease progression varied with the parameterization of the wind dispersal submodel (opening angle of the cone, wind direction in afternoon hours or for the hour where the speed was maximal etc.; results not shown). Results of a global sensitivity analysis in the model for Septoria tritici blotch (STB) in wheat (caused by Mycosphaerella graminicola) (Garin, 2015) suggested little impact of stem elongation rate on the disease severity, however the dispersal of STB was driven by rain splash. The rate of leaf and internode emergence, and senescence rate had the highest impact on disease severity, the sensitivity to leaf dimensions (length and width) was low (Garin, 2015).

There are many possibilities for model improvement and extension. Favourable temperatures in combination with continuous wetness are essential for the development of yellow spot (Rees and Platz, 1980; Francl, 1998; De Wolf et al., 1998). In the model, rainfall periods were required for ascospore maturation, and continuous leaf wetness, estimated by high relative humidity, for secondary infection. However, moist conditions affect other parts of disease cycle as well, e.g. release of ascospores or conidia production (Francl, 1998). To model these events in a continuous matter, additional response functions (model-scaling factors) could be used (cf. Audsley et al., 2005, Caubel et al., 2012). This would allow us to include information about optimal climatic conditions for maximal disease response. In the current model version, conidia are released at once after the end of latency, although Francl (1998) reported that only about $47 \%$ of conidia were liberated after 1 day. Additionally, the release is related to the weather conditions. The highest number of spores occured in dry afternoon hours (De Wolf et al., 1998), but the diurnal pattern differed with year and in early and late growing season (Francl, 1997). 
The next steps in model development will include sensitivity analysis to identify different sources of uncertainty related especially to the wind dispersal model and to improve parameterisation of the model. We will test the model applicability to predict disease progression in an artificially inoculated test canopy from field experiments. Dispersal gradients from field observations or a more realistic model for wind dispersal, which includes upward movement of wind and turbulent fluctuations, would benefit the accuracy of the model (Kuparinen, 2006). It would be interesting to study whether adding additional complexity into the wind dispersal model, e.g. by computing the trajectories of single spores, would better explain dispersal pattern observed in the field.

An advantage of using a mechanistic approach to model plant assimilation and allocation is that such model allows us to study in the future the potential response of the plant to the pathogen attack and the pathogen response to the induced changes in the plant. At this stage, the aim of the higher complexity of the model is to improve the understanding of various plant-pathogen-environment-management interactions and should help us to explain different observations. However, we will need to address model simplification and optimization issues (cf. Robert et al., 2008), e.g. if the model should be considered for application in disease prediction.

\section{ACKNOWLEDGEMENT}

This project was funded by Grains Research and Development Corporation (GRDC). We thank Christian Fournier, Christophe Pradal and Guillaume Garin for deep insight into pathogen modelling with FSPMs, Geoff Thomas, Manisha Shankar, Jean Galloway and Art Diggle for discussions on yellow spot development and modelling, and Darshan Sharma for discussions on wheat growth.

\section{REFERENCES}

Ando, K., Grumet, R., Terpstra, K., and Kelly, J.D. (2007). Manipulation of plant architecture to enhance crop disease control. CAB Reviews: Perspectives in Agriculture, Veterinary Science, Nutrition and Natural Resources, 2, No. 026.

Audsley, E., Milne, A., and Paveley, N. (2005). A foliar disease model for use in wheat disease management decision support systems. Annals of Applied Biology, 147, 161-172.

Calonnec, A., Cartolaro, P., Naulin, J.-M., Bailey, D., and Langlais, M. (2008). A host-pathogen simulation model: powdery mildew of grapevine. Plant Pathology, 57, 493-508.

Calonnec, A., Burie, J.-B., Langlais, M., Guyader, S., Saint-Jean, S., Sache, I., Tivoli, B. (2013). Impacts of plant growth and architecture on pathogen processes and their consequences for epidemic behavior. European Journal of Plant Pathology, 135, 479-497.

Caubel, J., Launay, M., Lannou, C., and Brisson, N. (2012). Generic response functions to simulate climate-based processes in models for the development of airborne fungal crop pathogens. Ecological Modelling, 242, 92104.

Chakraborty, S. and Newton, A.C. (2011). Climate change, plant diseases and food security: an overview. Plant Pathology, 60, 2-14.

De Wolf, E.D., Effertz, R.J., Ali, S., and Francl, L.J. (1998). Vistas of tan spot research. Canadian Journal of Plant Pathology, 20, 349-370.

De Wolf, E.D. and Francl, L.J. (1997). Neural Networks That Distinguish Infection Periods of Wheat Tan Spot in an Outdoor Environment. Phytopathology, 87, 83-87.

De Wolf, E.D. and Isard, S.A. (2007). Disease Cycle Approach to Plant Disease Prediction. Annual Review of Phytopathology, 45, 203-220.

Evers, J.B., Vos, J., Yin, X., Romero, P., van der Putten, P.E.L., and Struik, P.C. (2010). Simulation of wheat growth and development based on organ-level photosynthesis and assimilate allocation. Journal of Experimental Botany, 61, 2203-2216.

Evers, J.B. and Bastiaans, L. (2016). Quantifying the effect of crop spatial arrangement on weed suppression using functional-structural plant modelling. Journal of Plant Research, 129, 339-351.

Francl, L.J. (1997). Local and mesodistance dispersal of Pyrenophora tritici-repentis conidia. Canadian Journal of Plant Pathology, 19, 247-255.

Francl, L.J. (1998). Genesis and liberation of conidia of Pyrenophora tritici-repentis. Canadian Journal of Plant Pathology, 20, 387-393. 
Galloway, J., Beard, C., Payne, P., Thomas, G., Salam, M. (2017). Yellow spot of wheat: epidemiology studies and how they have helped us understand what we observe in the paddock. GRDC updates Goondiwindi.

Garin, G., Fournier, C., Andrieu, B., Houlès, V., Robert, C., and Pradal, C. (2014). A modelling framework to simulate foliar fungal epidemics using functional-structural plant models. Annals of Botany, 114, 795-812.

Garin, G. (2015). Vers la compréhension des épidémies fongiques foliaires par modélisation multi-échelle dans les couverts architectures. $\mathrm{PhD}$ thesis, AgroParisTech.

Goudriaan, J., van Laar, H.H. (1994). Modelling potential crop growth processes. Kluwer Academic Publishers, Dordrecht.

Hemmerling, R., Kniemeyer, O., Lanwert, D., Kurth, W., and Buck-Sorlin, G. (2008). The rule-based language $\mathrm{XL}$ and the modelling environment GroIMP illustrated with simulated tree competition. Functional Plant Biology, 35, 739-750.

Kuparinen, A. (2006). Mechanistic models for wind dispersal. TRENDS in Plant Science, 11, 296-301.

Landry, C., Bonnot, F., Ravigné, V., Carlier, J., Rengifo, D., Vaillant, J., and Abadie, C. (2017). A foliar disease simulation model to assist the design of new control methods against black leaf streak disease of banana. Ecological Modelling, 359, 383-397.

Marcelis, L.F.M. (1996). Sink strength as a determinant of dry matter partitioning in the whole plant. Journal of Experimental Botany, 47, 1281-1291.

Murray, G.M. and Brennan, J.P. (2009). The Current and Potential Costs from Diseases of Wheat in Australia. Grain Research and Development Corporation.

Pangga, I.B., Hanan, J., and Chakraborty, S. (2011). Pathogen dynamics in a crop canopy and their evolution under changing climate. Plant Pathology, 60, 70-81.

Rees, R.G., Platz, G.J. (1980). The Epidemiology of Yellow Spot of Wheat in Southern Queensland. Australian. Journal of Agricultural Research, 31, 259-267.

Salam, M.U., Khangura, R.K., Diggle, A.J., and Barbetti, M.J. (2003). Blackleg Sporacle: A Model for Predicting Onset of Pseudothecia Maturity and Seasonal Ascospore Showers in Relation to Blackleg of Canola. Phytopathology, 93, 1073-1081.

Summerell, B.A. and Burgess, L.W. (1988). Factors influencing production of pseudothecia by Pyrenophora tritici-repentis. Transactions of the British Mycological Society, 90, 557-562.

Wilson, P.A., Chakraborty, S. (1998). The virtual plant: a new tool for the study and management of plant diseases. Crop Protection, 17, 231-239.

Yin, X., Goudriaan, J., Lantinga, E.A., Vos, J., and Spiertz, H.J. (2003). A flexible sigmoid function of determinate growth. Annals of Botany, 91, 361-371. 